\title{
Direct measurement of the coherence length of edge states in the Integer Quantum Hall Regime
}

\author{
Preden Roulleau, F. Portier, and P. Roche* \\ Nanoelectronic group, Service de Physique de l'Etat Condensé, \\ CEA Saclay, F-91191 Gif-Sur-Yvette, France \\ A. Cavanna, G. Faini, U. Gennser, and D. Mailly \\ CNRS, Phynano team, \\ Laboratoire de Photonique et Nanostructures, \\ Route de Nozay, F-91460 Marcoussis, France
}

(Dated: November 5, 2018)

\begin{abstract}
We have determined the finite temperature coherence length of edge states in the Integer Quantum Hall Effect (IQHE) regime. This was realized by measuring the visibility of electronic Mach-Zehnder interferometers of different sizes, at filling factor 2. The visibility shows an exponential decay with the temperature. The characteristic temperature scale is found inversely proportional to the length of the interferometer arm, allowing to define a coherence length $l_{\varphi}$. The variations of $l_{\varphi}$ with magnetic field are the same for all samples, with a maximum located at the upper end of the quantum hall plateau. Our results provide the first accurate determination of $l_{\varphi}$ in the quantum Hall regime.
\end{abstract}

PACS numbers: 03.65.Yz, 73.43.Fj, 73.23.Ad

The understanding of the decoherence process is a major issue in solid state physics, especially in view of controlling entangled states for quantum information purposes. The edge states of the quantum Hall effect are known to present an extremely long coherence length $l_{\varphi}$ at low temperature [1], providing a useful tool for quantum interference experiments [2, 3, 4, 5, 6]. Surprisingly, very little is known on the exact value of this length and the mechanisms which reduce the coherence of edge states. This is in strong contrast with diffusive conductors, where weak localisation gives a powerful way to probe $l_{\varphi}$. It has been shown, in this case, that electronelectron interactions are responsible for the finite coherence length at low temperatures. In the IQHE regime, the presence of a high magnetic field destroys any time reversal symmetry needed for weak localisation corrections, making such an investigation difficult. Furthermore, due to the uni-dimensionality of the edge states, electron-electron interactions may strongly modify the single particle picture and one can ask wether the notion of phase coherence length is still relevant and how it depends on temperature. In this letter, we show for the first time that one can define a phase coherence length, and that it is inversely proportional to the temperature.

Though the energy redistribution length has been studied in the past [7, 8], these scattering experiments do not measure the phase coherence, which requires observation of electron interference effects. So far, experiments have only been able to put a lower bound on $l_{\varphi}$ at low temperatures [2, 9, 10, 11]. The electronic Fabry-Pérot interferences occurring in ballistic quantum dots have been used since the early days of mesoscopic physics [9]. These first studies showed an exponential decay of the amplitude of the Aharonov-Bohm (AB) oscillations with temperature [10]. However, this decay was attributed to thermal smearing due to the contribution of thermally activated one particle energy levels of the dot. Furthermore, the size of the interferometers was not varied, nor was a Fourier analysis performed of the $A B$ oscillations that could yield an estimation of $l_{\varphi}$ [12]. Quantum dot systems also implicate the possible interplay of Coulomb Blockade effects [13]. The Mach-Zender interferometers (MZI) 2, 4, 6] used in the present study do not suffer from the same limitations. First, we will show that the observed oscillations result from the interference of two paths of equal length, making thermal smearing negligible. Second, charge quantization effect leading to Coulomb blockade are irrelevant here. Last, comparison between interferometers of various sizes allows us the unambiguous determination of $l_{\varphi}$, as well as its dependence with temperature and magnetic field.

The sample geometry, presented in Fig. (1), is the same as in [6]. MZIs of different sizes were patterned using e-beam lithography on a high mobility two dimensional electron gas formed at the GaAs/ $\mathrm{Ga}_{1-x} \mathrm{Al}_{x} \mathrm{As}$ heterojunction (sheet density $n_{S}=2.0 \times 10^{11} \mathrm{~cm}^{-2}$ and mobility $\left.=2.5 \times 10^{6} \mathrm{~cm}^{2} / \mathrm{Vs}\right)$. The experiments were performed in the IQHE regime at filling factor $\nu=n_{S} h / e B=2$ (magnetic field $B \simeq 4.6$ Tesla). Transport occurs through two edge states. Quantum point contacts (QPC) G0, G1 and G2 define electronic beam splitters with transmissions $\mathcal{T}_{i}(\mathrm{i}=0-2)$. In all the results presented here, the interferences were studied on the outer edge state schematically drawn as red lines in Fig.(11), the inner edge state being fully reflected by all the QPCs. The first gate G0 is tuned to fully transmit the outer $\left(\mathcal{T}_{0}=1\right)$ edge state. The interferometer itself consists of G1, G2 and the small central ohmic contact 

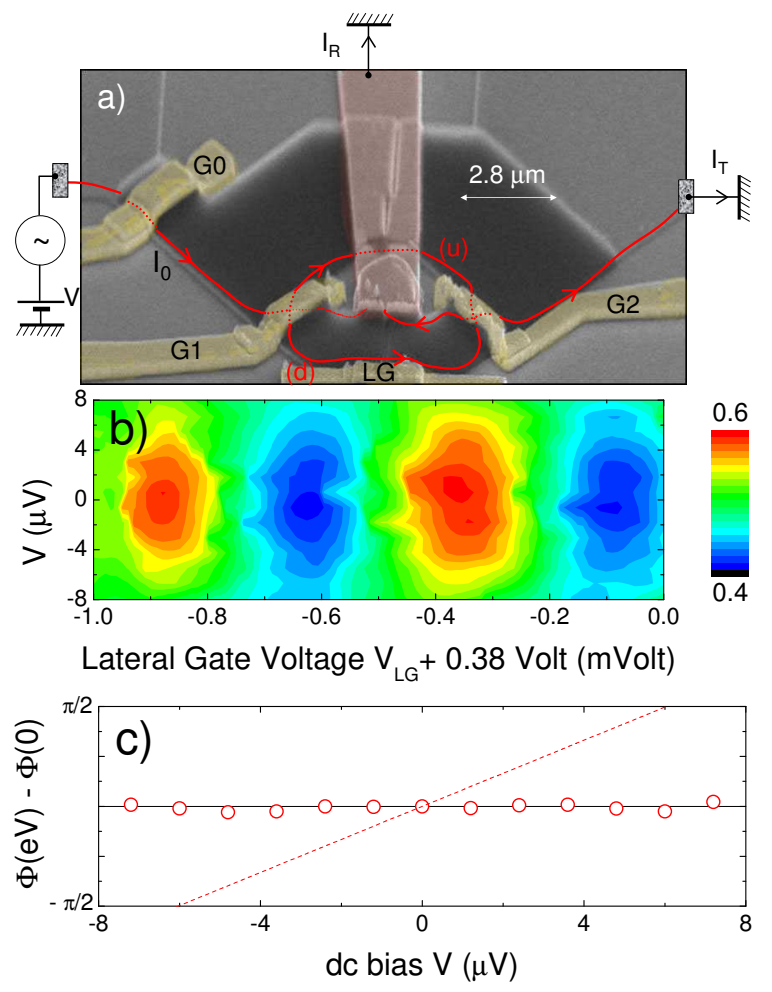

FIG. 1: (color online) a) Tilted SEM view of the "small" MZI. G0, G1 and G2 are QPCs whose split gates are connected with gold bridges over an isolator responsible for the black color of the SEM view. LG is a lateral gate which allows to vary the surface defined by the two arms (u) and (d) of the MZI. The interferences are measured in the IQHE at filling factor 2 on the outer edge state represented by a line on the SEM picture. The inner edge state is fully reflected by all the QPCs. The small ohmic contact in between the two arms collects the back scattered current $I_{B}$ to the ground through a long gold bridge. b) $2 \mathrm{D}$ plot of $d I_{T} / d I_{0}$ as a function of the lateral gate voltage $V_{L G}$ and the DC bias $V$, for the large sample at $20 \mathrm{mK}$. The visibility of interferences of the order of $20 \%-40 \%$ decreases with $V$ while the phase of interferences remains almost constant. c) Phase of the large sample deduced from Fig. (1b). The dashed line is the energy dependence of the phase which would be necessary to explain our observed visibility decrease with thermal smearing (see text).

in between the two arms. G1 splits the incident beam into two trajectories $(\mathrm{u})$ and $(\mathrm{d})$, which are recombined with G2, leading to interferences. Samples have been designed such that $(\mathrm{u})$ and $(\mathrm{d})$ are of equal length. The sizes of the three interferometers used in this study scale by up a factor $\sqrt{2}$ : the length of their arms are $\mathrm{L}=$ $5.6 \mu \mathrm{m}, 8 \mu \mathrm{m}$ and $11.3 \mu \mathrm{m}$ for enclosed areas of $8.5 \mu \mathrm{m}^{2}$ (referred to as 'small'), $17 \mu \mathrm{m}^{2}$ ('medium') and $34 \mu \mathrm{m}^{2}$ ('large'), respectively. The samples are cooled in a dilution refrigerator to temperatures ranging from $20 \mathrm{mK}$ to $\sim 200 \mathrm{mK}$.

The labels are indicated in the upper part of Fig. (1). A current $I_{0}$ is injected into the outer edge state through
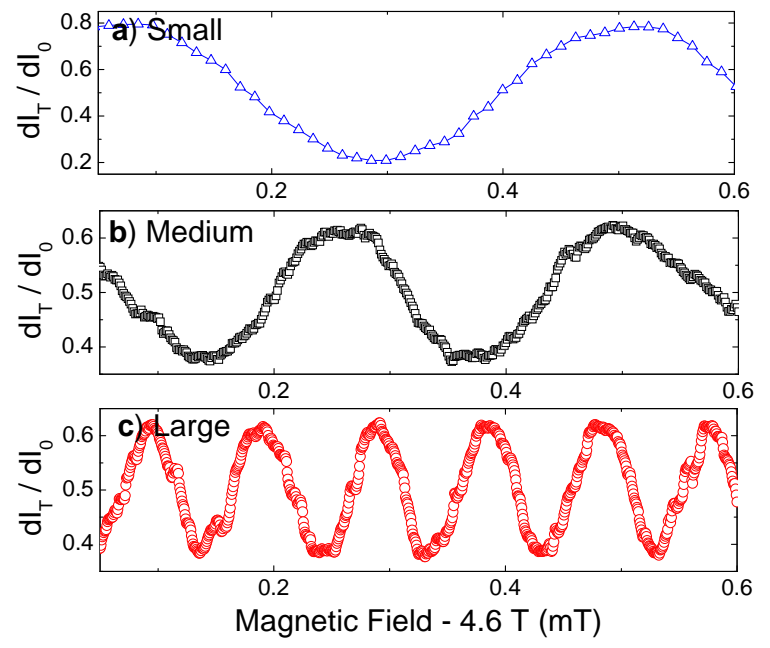

FIG. 2: (color online) Interferences revealed upon varying the magnetic flux through the surface defined by the two arms $(\mathrm{u})$ and $(d)$ of the interferometers. From the oscillation period $\delta B$ we deduce the surface $S=h /(e \delta B)$ of the 3 different studied MZI. a) The small MZI $\left(S=8.7 \pm 0.2 \mu \mathrm{m}^{2}\right)$. b) The medium MZI $\left(S=15.5 \pm 0.4 \mu \mathrm{m}^{2}\right)$. c) The large MZI $(S=$ $\left.40.7 \pm 0.8 \mu \mathrm{m}^{2}\right)$. All these surfaces are in good agreement with the lithographic ones (see text).

the interferometer. The current which is not transmitted, $I_{B}=I_{0}-I_{T}$, is collected to the ground with the small central ohmic contact. $I_{0}$ is made up of a minute AC part, with the possibility to superimpose a DC bias $V$. The differential transmission of the interferometer is defined as $\mathcal{T}=G / G_{0}=d I_{T} / d I_{0}$, where $G=d I_{T} / d V$ is the differential conductance and $G_{0}=e^{2} / h$. It is measured with a standard lock-in technique using a $619 \mathrm{~Hz}$ frequency and a $39 \mathrm{pA}_{r m s}$ amplitude AC bias. The corresponding bias voltage excitation $\left(1 \mu \mathrm{V}_{r m s}\right)$ is always smaller than the energy scale involved. The oscillations revealing the quantum interferences can be obtained using two equivalent experimental procedures: either by superimposing a minute current to the large current of the magnet, or by changing the surface defined by the MZI using a lateral gate. Fig. (2) shows the AB oscillations of the transmission for the three interferometers, showing a magnetic period inversely proportional to the area of the interferometer, while Fig. (1b) shows oscillations obtained using the lateral gate LG. After checking that both methods lead to the same interferences amplitude, we have always used the lateral gate and run the magnet in the permanent-current mode, strongly reducing the measurement noise. The visibility of the AB oscillations is defined as the ratio of the half amplitude of the oscillation of the transmission divided by the mean value, $\mathcal{V}=\left(\mathcal{T}_{\max }-\mathcal{T}_{\min }\right) /\left(\mathcal{T}_{\max }+\mathcal{T}_{\text {min }}\right)$

The maximum value of $\mathcal{V}$ is always obtained at the lowest temperature. $\mathcal{V}$ can reach $65 \%$ for the small interferometer, whereas it typically attains $20-40 \%$ for the medium and the large interferometers (see Fig. (1b)). 


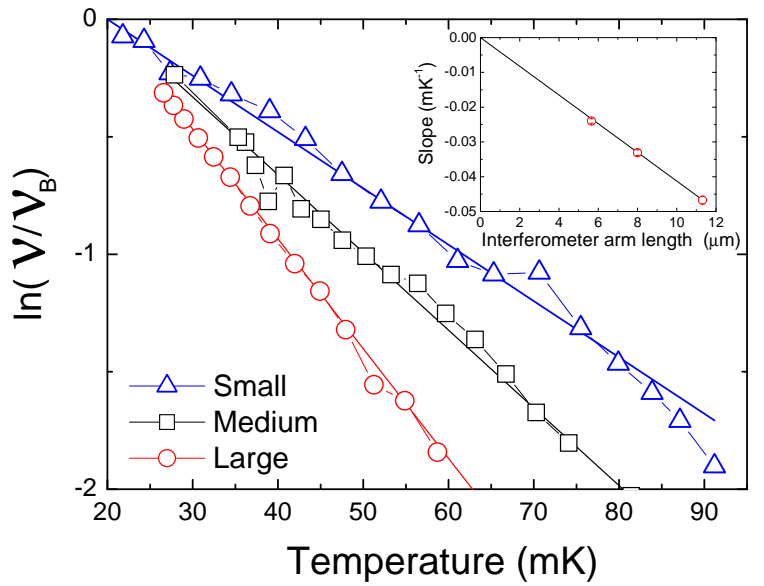

FIG. 3: (color online) $\ln \left(\mathcal{V} / \mathcal{V}_{B}\right)$ as a function of the temperature for the 3 different samples, $\mathcal{V}_{B}$ being the visibility measured at $T_{B}=20 \mathrm{mK}$. The measurement has been done at the magnetic field for which the visibility decay is the smallest. Inset : The slope $\ln \left(\mathcal{V} / \mathcal{V}_{B}\right) /\left(T-T_{B}\right)$ is proportional to the arm length.

For each interferometer we have studied the temperature dependence of the visibility. In fig. (3), we have plotted $\ln \left(\mathcal{V} / \mathcal{V}_{B}\right)$ versus temperature, where $\mathcal{V}_{B}$ stands for the visibility at $T_{B}=20 \mathrm{mK}$. Clearly, the visibility decreases with temperature in all cases, and the larger the interferometer, the stronger the temperature dependence. More quantitatively, if a linear regression of $\ln \left(\mathcal{V} / \mathcal{V}_{B}\right)=\left(T-T_{B}\right) / T_{0}$ is done, one finds that $T_{0}^{-1}$ is proportional to the length of the interfering arms (inset of Fig. (3) ). In the following, we show that this behavior does not result from a thermal smearing.

The transmission probability through the MZI at the energy $\epsilon$ is $\mathcal{T}(\epsilon)=\mathcal{T}_{1} \mathcal{T}_{2}+\mathcal{R}_{1} \mathcal{R}_{2}+z \sqrt{\mathcal{T}_{1} \mathcal{R}_{2} \mathcal{R}_{1} \mathcal{T}_{2}} \sin [\phi(\epsilon)]$, where $z \in[0,1]$ is a parameter accounting for phase averaging and/or decoherence, and $\mathcal{T}_{i}=\left|t_{i}\right|^{2}=1-\mathcal{R}_{i}$ are the beam splitters transmissions [14, 15]. $\phi(\epsilon)$ is the AB flux across the surface $S(\epsilon)$ defined by the energy dependent edge state positions in the two interfering arms, $\phi(\epsilon)=2 \pi S(\epsilon) \times e B / h$. When there is a finite length difference $\Delta L=L_{u}-L_{d}$ between the two arms, the surface $S$ depends on the energy $\epsilon$. Thus the phase varies with the energy, $\phi\left(\epsilon+E_{F}\right)=\phi\left(E_{F}\right)+\epsilon /\left(k_{B} T_{S}\right)$ where $k_{B} T_{S}=\hbar v_{D} / \Delta L$ [15] and $v_{D}$ is the drift velocity $\left(10^{4}\right.$ to $\left.10^{5} \mathrm{~ms}^{-1}\right)$ 16]. The differential conductance $G$ at bias $V$ and at temperature $T$ probes the transmission probability at energy $\mathrm{eV}$ smeared over an energy range $k_{B} T$ [15]: $G(V)=G_{0} \int_{-\infty}^{+\infty} f(\epsilon) \mathcal{T}(\epsilon+e V) \mathrm{d} \epsilon \propto\left[1+\mathcal{V}_{0}<\right.$ $\left.\sin \left(\phi(e V)>k_{B} T\right)\right]$, where $f(\epsilon)$ is the Fermi distribution at temperature $T$. The energy dependence of the phase $\phi$ leads to a thermal smearing at finite temperature as the phase is blurred. A complete calculation yields a visibility decreasing like $\mathcal{V}=\mathcal{V}_{0} \pi T /\left(\pi T_{S} \sinh \left(\pi T / T_{S}\right)\right)[15$. At low temperature, $T_{S}$ can be determined by measuring the phase of the interferences as a function of the dc bias
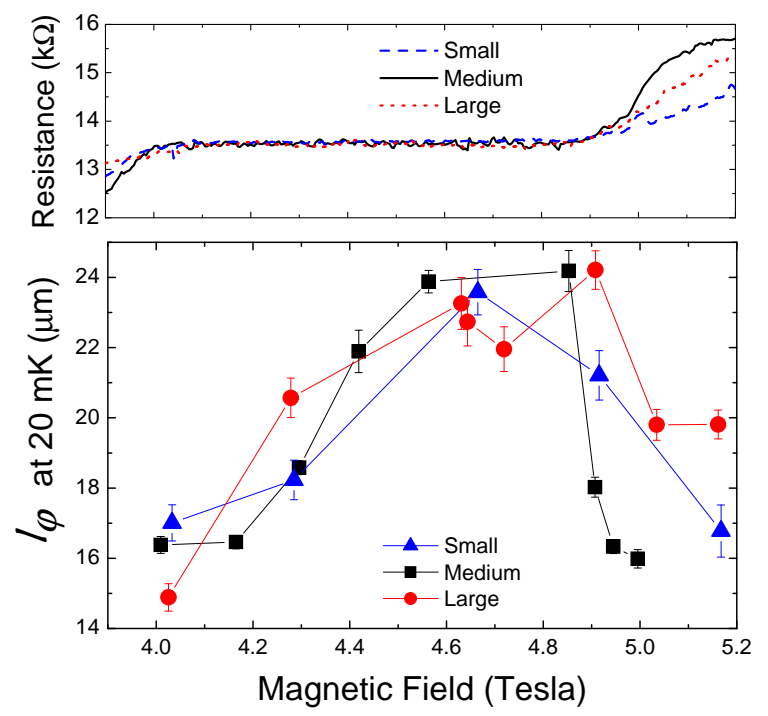

FIG. 4: (color online) Upper panel : The dashed, solid and dot lines are the two point Hall resistance at filling factor 2 measured for the small, the medium and the large sample respectively. $l_{\varphi}$ has a general shape recovered by all the three samples, with a maximum at the end of the Hall plateau. Lower panel : Coherence length at $20 \mathrm{mK}$ deduced from $L \times\left(T-T_{B}\right) / \ln \left(\mathcal{V} / \mathcal{V}_{B}\right)$ for the three different samples studied $(L=5.6,8$ and $11.3 \mu \mathrm{m})$. The magnetic fields (x-axis) of the small and large sample has been shifted by +0.25 and -0.1 Tesla respectively, such that the plateau centers coincide for the three samples.

\section{$V: \phi(e V)=\phi(0)+e V /\left(k_{B} T_{S}\right)$.}

In order to fit the visibility decrease with thermal smearing, this requires that $T_{S} \sim 66,59$ and $44 \mathrm{mK}$, for the small, medium and large sample respectively. In Fig. (1b) we have plotted a $2 \mathrm{D}$ graph of the differential transmission $\mathcal{T}(V)$ as a function of the lateral gate voltage and the dc bias, for the large sample at $20 \mathrm{mK}$. From this measurement we have deduced the phase $\phi(e \mathrm{~V})$ which is shown to remain almost constant over an energy range of $\sim 16 \mu \mathrm{eV}$ (Fig. (1c)). As a comparison, the dashed line of (Fig. (1c)) is the phase dependence which would be required $\left(T_{S}=44 \mathrm{mK}\right)$ to explain the decrease of the visibility with thermal smearing. The conclusion is straightforward: our sample does not suffer from thermal smearing. We have done the same procedure for all the three samples which exhibits a phase rigidity over at least $\sim 16 \mu \mathrm{eV}$, meaning that all our samples have negligible thermal smearing in the explored temperature range $k_{B} T<16 \mu \mathrm{V} \equiv 200 \mathrm{mK}$.

The exponential decrease of the visibility with temperature is robust against various parameter variations, revealing a universal behaviour. While the maximum visibility at the lowest temperature is affected by varying the transmissions $\mathcal{T}_{i}$ of the MZI and by applying a finite bias [6], the slope $\ln \left(\mathcal{V} / \mathcal{V}_{B}\right) /\left(T-T_{B}\right)$ is found to be unaffected.

Indeed, and this is the central result of our paper, our 
measurements can be interpreted by the introduction of a coherence length $l_{\varphi}(T)$ such that

$$
\mathcal{V}=\mathcal{V}_{0} e^{-2 L / l_{\varphi}} \text { with } l_{\varphi} \propto T^{-1}
$$

as shown in Fig. (3). $\mathcal{V}_{0}$ contains the temperature independent part of the visibility. In the inset of the Fig. (3), we have plotted the slope $\ln \left(\mathcal{V} / \mathcal{V}_{B}\right) /\left(T-T_{B}\right)$ for the three samples [20]. It is clear that the slope scales with the length of the interferometer arm defining, de facto, a coherence length $l_{\varphi}(T)$ of about $20 \mu \mathrm{m}$ at $20 \mathrm{mK}$. The magnetic field variation of the deduced $l_{\varphi}$ is independent from the MZI size (see Fig. (4D)). In order to compare the three samples at the same filling factor, we have shifted the $\mathrm{x}$-axis of Fig. (4) by $+0.25 \mathrm{~T}$ and $-0.1 \mathrm{~T}$ for the small and large MZI respectively. These values center the Hall plateaus all together. The maximum of the coherence length is reached at the upper end of the plateau where the longitudinal resistance is usually minimum. However, our sample configuration does not allow us to check if it is actually the case.

Let us now compare our results with previously available data from other groups. Although the variations of $\mathcal{V}(T)$ were measured only for one interferometer size in the following experiments, it is possible to fit the data with equation 1 and to deduce a coherence length value at $20 \mathrm{mK}$. In the Fabry-Pérot type interferometer (Fig. (5b) of ref.[10]), our analysis, using a coherence length instead of thermal smearing, leads to $l_{\varphi} \sim 20 \mu \mathrm{m}$ at $20 \mathrm{mK}$. Although the experiments of ref.[10] were performed with different filling factor, magnetic field, mobility, density and geometry, surprisingly it gives the same result. The data from ref. 2] yields also a similar $l_{\varphi}$, although a direct comparison is difficult without an exact knowledge of the MZI dimensions. Finally, the results of ref. [4], again interpreted by the authors as resulting from thermal smearing, lead to $l_{\varphi} \sim 80 \mu \mathrm{m}$ at $20 \mathrm{mK}$.

What kind of mechanism is responsible for a finite coherence length varying with a $T^{-1}$ temperature dependence? Electron - electron collisions are known to limit the coherence in non unidimensional conductors $(2 \mathrm{D}$ electron gas, diffusive metallic conductors ...). For the MZI, a finite $l_{\varphi}$ coming from either short range interaction $\left(l_{\varphi} \propto T^{-3}\right)$, long range interaction $\left(l_{\varphi} \propto T^{-1} \ln ^{2}(1 / T)\right)$ or curvature of the fermion dispersion $\left(l_{\varphi} \propto T^{-2}\right)[17$ ] cannot explain our findings. Alternatively, interactions with environment electrons, capacitively coupled to the arms of the interferometer, have been proposed to describe the decoherence of MZIs [18]. More specifically, decoherence is due to the thermal noise of the dissipative part of the finite frequency coupling impedance between the environment and the reservoirs. This theory leads to

$$
\frac{l_{\varphi}}{L}=\frac{\tau_{\varphi}}{\tau}=\frac{3 \hbar}{2 \pi k_{B} T} \frac{v_{D}}{L} \times\left(1+\frac{\pi \hbar C v_{D}}{L e^{2}}\right)^{2},
$$

up to a numerical factor decreasing from $3 / 2$ to 1 when $\pi \hbar C v_{D} /\left(L e^{2}\right)$ increases from 0 to $\infty$ (Eq. 37 of ref.[18]).
$C$ is a geometric capacitance, which represents the coupling to the environment and $\tau$ is the time of flight. For $v_{D}=5.10^{4} \mathrm{~ms}^{-1}$ and $C / L \sim \epsilon_{r} \epsilon_{0}$, one finds $\pi \hbar C v_{D} /\left(L e^{2}\right) \ll 1$ and $l_{\varphi} \sim 14 \mu \mathrm{m}$ at $20 \mathrm{mK}$. This result agrees with our measurements, although in the absence of an independent determination of $v_{D}$ and $C$, it is not possible to be more quantitative.

We now turn to the non monotonic dependence of $l_{\varphi}$ with the magnetic field $B$. If $\tau_{\varphi}$ is independent of $B$, as suggested by equation (2), the apparent variation of $l_{\varphi}$ results from a variation of $\tau$. As we have deduced $l_{\varphi}$ assuming a constant trajectory length $l=L$, any variation of $l$, due to disorder, would modify the deduced $l_{\varphi}=\tau_{\varphi} L \times v_{D} / l$. Then, the maximum of $l_{\varphi}$ shown in Fig. (4) corresponds to the minimum of $l / v_{D}$. In a naive picture, the drift velocity $v_{D}$ varies like $1 / B$ [16, 19] barely leading to a non monotonic variations of $l_{\varphi}$. In another hand, a non monotonic variation of $l$ is all the more plausible. The maximum of $l_{\varphi}$ occurs on the upper end of the Hall plateau where one expects minimum backscattering, thus a minimum $l$. Assuming this explanation correct, the overlap of the three curves in Fig. (4) (lower panel) indicates that the variations of $l$ scale with the geometric length of the MZI. The study of the influence of the sample disorder on the coherence length and its dependence with magnetic field could bring new insights supporting our assumption.

In conclusion, we have measured the visibility of MachZehnder interferometers of various sizes, operating in the IQHE regime at filling factor 2 , as a function of both the temperature and the magnetic field. Our results provide a direct and reliable measurement of the coherence length found to be inversely proportional to the temperature and maximum at the upper end of the Hall plateau. The order of magnitude is compatible with theoretical predictions based on a dephasing arising from the thermal noise of the environment.

* Electronic address: patrice.roche@cea.fr

[1] T. Martin and S. Feng, Phys. Rev. Lett. 64, 1971 (1990).

[2] Y. Ji et al., Nature 422, 415 (2003).

[3] P. Samuelsson, E. V. Sukhorukov, and M. Büttiker, Phys. Rev. Lett. 92, 026805 (2004).

[4] L. V. Litvin et al., Phys. Rev. B 75, 033315 (2007).

[5] I. Neder et al., Nature 448, 333 (2007).

[6] P. Roulleau et al., Phys. Rev. B 76, (2007).

[7] B. W. Alphenaar, P. L. MacEuen, R. G. Wheeler, and R. N. Sacks, Phys. Rev. Lett. 64, 677 (1990).

[8] T. Machida et al., Solid State Commun. 103, 441 (1997).

[9] B. J. van Wees et al., Phys. Rev. Lett. 62, 2523 (1989).

[10] J. P. Bird et al., Phys. Rev. B 50, 14983 (1994).

[11] I. Yang et al., Phys. Rev. B 71, 113312 (2005).

[12] A. E. Hansen et al., Phys. Rev. B 64, 045327 (2001).

[13] B. Rosenow and B. I. Halperin, Phys. Rev. Lett. 98, 106801 (2007). 
[14] F. Marquardt and C. Bruder, Phys. Rev. B 70, 125305 (2004).

[15] V. S.-W. Chung, P. Samuelsson, and M. Büttiker, Phys. Rev. B 72, 125320 (2005).

[16] R. C. Ashoori et al., Phys. Rev. B 45, R3894 (1992).

[17] J. T. Chalkers, Y. Gefen, and M. Y. Veillette, Phys. Rev. B 76, 085320 (2007).
[18] G. Seelig and M. Buttiker, Phys. Rev. B 64, 245313 (2001).

[19] C. W. J. Beenakker, Phys. Rev. Lett. 64, 216 (1990).

[20] These slopes are the minimum values obtained at different magnetic fields. From small to large MZI: 4.41 T, 4.91 $\mathrm{T}$ and $4.85 \mathrm{~T}$. 